\title{
DISCONTINUOUS ROBUST MAPPINGS ARE APPROXIMATABLE
}

\author{
SHUZHONG SHI, QUAN ZHENG AND DEMING ZHUANG
}

\begin{abstract}
The concepts of robustness of sets and and functions were introduced to form the foundation of the theory of integral global optimization. A set $A$ of a topological space $X$ is said to be robust iff $\mathrm{cl} A=\operatorname{cl}$ int $A$. A mapping $f: X \rightarrow Y$ is said to be robust iff for each open set $U_{Y}$ of $Y, f^{-1}\left(U_{Y}\right)$ is robust. We prove that if $X$ is a Baire space and $Y$ satisfies the second axiom of countability, then a mapping $f: X \rightarrow Y$ is robust iff it is approximatable in the sense that the set of points of continuity of $f$ is dense in $X$ and that for any other point $x \in X,(x, f(x))$ is the limit of $\left\{\left(x_{\alpha}, f\left(x_{\alpha}\right)\right)\right\}$, where for all $\alpha, x_{\alpha}$ is a continuous point of $f$. This result justifies the notion of robustness.
\end{abstract}

\section{INTRODUCTION}

The concepts of robustness of sets and functions were proposed in [10]-[12] for establishing the theory of integral global optimization and for weakening the requirement of continuity of the objective function in the global optimization problems. Until now, the importance of this concept has not been sufficiently addressed and affirmed. This paper will show that the nature of a discontinuous robust mapping is its "approximatability", which is the essence of numerical analysis.

The main goals of numerical analysis are to provide effective approximation procedures for solving equations, which may be algebraic, differential or those deduced from optimization, calculus of variations, optimal control and so on. A general problem of solving equations may be formulated as follows:

$$
\text { to find } \bar{x} \in X \text { such that } f(\bar{x})=\bar{y} \text {, }
$$

where $X$ and $Y$ are two sets, $f: X \rightarrow Y$ is a map and $\bar{y} \in Y$ is given. An effective approximation procedure or an algorithm for solving this equation is a rule of constructing a sequence $\left\{x_{k}\right\}$ such that $x_{k} \rightarrow \bar{x}$ and $y_{k}=f\left(x_{k}\right) \rightarrow \bar{y}$. Therefore, $X$ and $Y$ should be two topological spaces in order that the two limits are well defined; and usually $f$ is assumed to be continuous at $x=\bar{x}$. Surely, if $f(\bar{x})$ is not assumed to be related in any way to the values of $f$ near

Received by the editors March 26, 1992 and, in revised form, November 29, 1994.

1991 Mathematics Subject Classification. Primary 49J45, 54C08; Secondary 90C48.

Key words and phrases. Robust sets, robust mappings, approximatable mappings, integral global optimization.

Research of the first author supported partially by National Natural Science Foundation of China.

Research of the third author supported partially by NSERC grant and Mount St. Vincent University research grant. 
$\bar{x}$, it is impossible to propose an algorithm to produce an approximate solution to the equation (1). For example, suppose that $X=Y=\mathbf{R}, \bar{y}=0$ and

$$
f(x)= \begin{cases}1 & \text { if } x \neq 0 \\ 0 & \text { if } x=0\end{cases}
$$

Then the solution $\bar{x}=0$ cannot be approximated by using the value of $f$ near $\bar{x}$.

However, many problems from both theory and applications do require solving an equation of a discontinuous mapping. Examples include the likelihood functions and the expected utility functions when the probability distributions possess discontinuities. For such examples one often attempts to reduce the problem to one of continuous mappings. For instance, there exist several works ([1], [5], [9]), in which minimization problems of discontinuous functions are treated by combining smoothing techniques with conventional optimization methods. But these methods are complicated.

In our opinion, the continuity of $f$ is not indispensable for designing an algorithm to solve the equation (1). A more reasonable concept for numerical analysis should be the approximation. In fact, many discontinuous mappings are "approximatable". We consider the following examples:

Example 1.1. $X=Y=\mathbf{R}, \bar{y}=0$ and

$$
f(x)= \begin{cases}1 & \text { if } x<0 \\ x & \text { if } x \geq 0\end{cases}
$$

Example 1.2. $X=Y=\mathbf{R}, \bar{y}=0$ and

$$
f(x)= \begin{cases}1 & \text { if } \sin (1 / x)<0 \\ \sin (1 / x) & \text { if } \sin (1 / x) \geq 0 \\ 0 & \text { if } x=0\end{cases}
$$

In Example 1.1, $f$ is only right-continuous at $\bar{x}=0$; in Example 1.2, $f$ is not even one-sidedly continuous at $\bar{x}=0$. Nevertheless, for these two functions, the approximation to $\bar{x}$ is possible, because there always exists a sequence $\left\{x_{k}\right\}$ of points of continuity of $f$ such that

$$
\lim _{k \rightarrow \infty} x_{k}=\bar{x} \text { and } \lim _{k \rightarrow \infty} f\left(x_{k}\right)=f(\bar{x})
$$

and it is possible to approximate points of continuity of $f$.

It is natural to define an approximatable mapping $f: X \rightarrow Y$ by the approximatability of all the points $(x, f(x))$ in the graph of $f$. The first aim of this paper is to prove that any approximatable mapping is robust, and that under some mild hypotheses, the converse is also valid. This amounts to saying that in a suitable setting the concept of robust mappings is actually equivalent to that of approximatable mappings. This affirmation greatly justifies the notion of robustness.

The outline of the paper is as follows. We first give the definitions of robust mappings and of approximatable mappings (Section 2). In Section 3, we prove our main theorem: a robust mapping is approximatable under the conditions that $X$ is a Baire space and $Y$ satisfies the second axiom of countability. In 
Section 4, we suppose that $X$ is a complete metric space and $Y$ is a real separable Banach space and show that all bounded robust (approximatable) mappings from $X$ to $Y$ can be divided into many "Banach spaces of bounded robust (approximatable) mappings", the intersection of which is the Banach space of bounded continuous mappings. In Section 5 , we discuss the robust functions, which were stated by Zheng [10]-[13] and Zheng-Zhuang [15], but only for the upper topology on $\mathbf{R}$. Our main theorem implies that a robust function for the natural topology on $\mathbf{R}$ is just an approximatable function. However, we can also prove that any "upper robust function" (i.e. robust function in the sense of [10]-[13]) has the dense set of points of continuity. Section 6 contains some concluding remarks.

\section{ROBUST MAPPINGS AND APPROXIMATABLE MAPPINGS}

The concept of robust sets was proposed in [10]-[12]. We recall the definition.

Definition 2.1. Let $X$ be a topological space and $A$ be a subset of $X . A$ is said to be robust iff

$$
\operatorname{cl} A=\operatorname{cl} \text { int } A \text {, }
$$

where $\operatorname{cl} A$ is the closure of $A$ and int $A$ is the interior of $A$. A point $x \in A$ is said to be a robust point of $A$ iff $x \in \mathrm{cl}$ int $A$. If $x$ is a robust point of $A$, then $A$ is said to be a semi-neighbourhood of $x$.

The concepts of robust sets, robust points and semi-neighbourhoods are extensions of those of open sets, interior points and neighbourhoods, respectively. Any open set of $X$, including the empty set, is robust. Any interior point of $A$ is a robust point of $A$. Any neighbourhood of $x$ is a semi-neighbourhood of $x$. A set $A \subset X$ is robust iff all its points are robust points of $A$ or $A$ is a semi-neighbourhood of all its points. If $A$ is a semi-neighbourhood of $x$ and $A \subset B$, then $B$ is also a semi-neighbourhood of $x$.

Any union of robust sets is robust, but the intersection of two robust sets may not be robust unless one of them is open. Similarly, any union of semineighbourhoods of $x$ is also a semi-neighbourhood of $A$, but the intersection of two semi-neighbourhoods of $x$ may not be a semi-neighbourhood of $x$ unless one of them is a neighbourhood of $x$.

Recall that a set $A \subset X$ is nowhere dense iff int $\mathrm{cl} A=\varnothing$. Then we have

Proposition 2.1. Any robust set or its complement can be represented by the union of an open set and a nowhere dense set.

Proof. From the definition (5), for a robust set $A$, int $A$ and $A$ have the same boundary: $\partial A=\partial$ int $A$, which is nowhere dense. Obviously, $A$ is the union of int $A$ and a set $F \subset \partial A$. The complement of $A$ is the union of the complement of $\mathrm{cl} A$ and $\partial A \backslash F$.

Remark. Recall ([6], [7]) that a closed (respectively, open) set $A \subset X$ is said to be regular iff $A=\mathrm{cl}$ int $A$ (respectively, $A=$ int $\mathrm{cl} A$ ). The complement of a regular closed set is a regular open set and vice versa. By Definition 2.1, $A \subset X$ is robust iff its closure is regular or the interior of its complement is regular.

A set $A \subset X$ is said to be of the first category iff it is the union of a countable family of nowhere dense sets. A set $A \subset X$ is said to be a $G_{\delta}$ set iff it is the intersection of a countable family of open sets. A set $A \subset X$ is said to be an $F_{\sigma}$ 
set iff it is the union of a countable family of closed sets. A set $A \subset X$ has the Baire property iff it can be represented as a $G_{\delta}$ set plus (or an $F_{\sigma}$ set minus) a set of the first category. By Proposition 2.1, any robust set and its complement have the Baire property.

Definition 2.2. Let $X$ and $Y$ be two topological spaces and $f: X \rightarrow Y$ be a mapping. $f$ is said to be robust at $x \in X$ iff for any neighbourhood $U_{Y}(y)$ of $y=f(x), f^{-1}\left(U_{Y}(y)\right)$ is a semi-neighbourhood of $x$ or $x$ is a robust point of $f^{-1}\left(U_{Y}(y)\right)$. $f$ is said to be robust iff $f$ is robust at every $x \in X$ or for any open set $U_{Y}$ of $Y, f^{-1}\left(U_{Y}\right)$ is robust in $X$.

The concept of robustness for a mapping is an extension of that of continuity. Any continuous mapping is robust, but a robust mapping may be discontinuous (see Example 1.1-2).

Remark. Recall ([6], [7]) that a mapping $f: X \rightarrow Y$ has the Baire property iff for any open set $U_{Y}$ of $Y, f^{-1}\left(U_{Y}\right)$ has the Baire property. By Definition 2.2, any robust mapping has the Baire property.

The following proposition shows that the uniform convergence preserves the robustness of mappings.

Proposition 2.2. Let $X$ be a topological space, $Y=(Y, d)$ be a metric space and $f_{\delta}: X \rightarrow Y, \delta \in \Delta$ be a net of mappings. Suppose that all $f_{\delta}$ are robust at $\bar{x} \in X$ and a mapping $f: X \rightarrow Y$ satisfies

$$
\lim _{\delta} \sup _{x \in X} d\left(f_{\delta}(x), f(x)\right)=0 .
$$

Then $f$ is also robust at $\bar{x}$.

Proof. We have to prove that for $\bar{x} \in X$ and for any $\varepsilon$-neighbourhood of $\bar{y}=f(\bar{x})$

$$
U_{\varepsilon}(\bar{y})=\{y \in Y \mid d(y, \bar{y})<\varepsilon\} \quad(\varepsilon>0),
$$

$f^{-1}\left(U_{\varepsilon}(\bar{y})\right)$ is a semi-neighbourhood of $\bar{x}$. It suffices to show that there exists a semi-neighborhood $V(\bar{x})$ of $\bar{x}$ such that

$$
\forall x \in V(\bar{x}), \quad d(f(x), f(\bar{x}))<\varepsilon .
$$

From (6), using standard $\varepsilon / 3$ argument, we can easily verify that (7) holds.

Now we propose the definition of an approximatable mapping.

Definition 2.3. Let $X$ and $Y$ be two topological spaces and $f: X \rightarrow Y$ be a mapping. Suppose that $S$ is the set of points of continuity of $f$. Then $f$ is said to be approximatable iff

1. $S$ is dense in $X$;

2. for any $\bar{x} \in X$, there exists a net $\left\{x_{\alpha}\right\} \subset S$ such that

$$
\lim _{\alpha} x_{\alpha}=\bar{x} \text { and } \lim _{\alpha} f\left(x_{\alpha}\right)=f(\bar{x}) \text {. }
$$

Theorem 2.1. Any approximatable mapping is robust.

Proof. Let $f: X \rightarrow Y$ be an approximatable mapping. We show that for any open set $U_{Y}$ of $Y, f^{-1}\left(U_{Y}\right)$ is a robust set of $X$. In fact, if $x \in f^{-1}\left(U_{Y}\right)$ is a continuous point of $f$, then $x$ must be an interior point of $f^{-1}\left(U_{Y}\right)$, because 
$f^{-1}\left(U_{Y}\right)$, as the inverse image of a neighbourhood of $f(x)$, is a neighbourhood of $x$. On the other hand, if $\bar{x} \in f^{-1}\left(U_{Y}\right)$ is not a continuous point of $f$, then by the definition of approximatable mapping, there exists a net $\left\{x_{\alpha}\right\} \subset S$ such that $\lim _{\alpha} x_{\alpha}=\bar{x}$ and $\lim _{\alpha} f\left(x_{\alpha}\right)=f(\bar{x})$, where every $x_{\alpha}$ is a continuous point of $f$. Since $U_{Y}$ is a neighbourhood of $f(\bar{x})$, for $\alpha$ sufficiently large, $f\left(x_{\alpha}\right) \in U_{Y}$, and then, $x_{\alpha}$ is an interior point of $f^{-1}\left(U_{Y}\right)$. Hence, $\bar{x}$ is a cluster point of the interior of $f^{-1}\left(U_{Y}\right)$. That is to say that $\bar{x}$ is a robust point of $f^{-1}\left(U_{Y}\right)$. Thus, $f^{-1}\left(U_{Y}\right)$ is a robust set of $X$.

The simplest example of a discontinuous approximatable mapping is a robust piecewise continuous mapping in the following sense:

Definition 2.4. Let $X$ and $Y$ be two topological spaces. A mapping $f: X \rightarrow Y$ is said to be robust piecewise continuous iff there exists a "robust partition" of $X$, i.e.

$$
X=\bigcup_{i \in I} V_{i} \text { and } \forall i \in I, \quad i \neq j, \quad V_{i} \cap V_{j}=\varnothing,
$$

where for any $i \in I, V_{i}$ is robust in $X$, and for any $i \in I$, the restriction of $f$ in $V_{i}$ is continuous.

Obviously, when $Y$ is a metric space (even a uniform space), the uniform convergence also preserves the robust piecewise continuity of mappings. Notice that if in its definition the partition of $X$ is not required to be "robust", then a piecewise continuous mapping may not be robust.

A mapping whose points of continuity form a dense subset of the domain may not be approximatable.

Example 2.1. Let $X=Y=\mathbf{R}$ and

$$
f(x)= \begin{cases}\sin (1 / x) & \text { if } x \neq 0 \\ \alpha & \text { if } x=0\end{cases}
$$

Then $f$ is approximatable iff $\alpha \in[-1,1]$.

An approximatable mapping can possess a dense set of points of discontinuity as illustrated in the following example.

Example 2.2. Let $X=Y=[0,1],\left\{r_{k}\right\}$ be the set of all rational numbers in $[0,1]$ and $\alpha_{k}>0, k=1,2, \ldots$, with $\sum_{k=1}^{\infty} \alpha_{k}=1$. Then the function

$$
f(x)=\sum_{r_{k}<x} \alpha_{k}
$$

is a left-continuous monotone function from $[0,1]$ to $[0,1]$ and discontinuous at every rational point in $[0,1]$.

\section{MAIN THEOREM}

Recall that a topological space is said to be a Baire space iff no nonempty open set in the space is of the first category; a topological space is said to satisfy the second axiom of countability iff it has a countable base (of open sets). Then we have 
Theorem 3.1. Assume that $X$ is a Baire space and $Y$ satisfies the second axiom of countability. Then a mapping $f: X \rightarrow Y$ is robust iff it is approximatable. In this case, the set $D$ of points of discontinuity of a robust mapping is always of the first category. In addition, if $Y$ is a separable metric space, then $D$ is an $F_{\sigma}$ set of the first category.

The proof of this theorem is based on the following four propositions.

Proposition 3.1 [4]. Let $X$ and $Y$ be two topological spaces and $f: X \rightarrow Y$ be a mapping. Assume that $Y$ has a base $\left\{U_{\alpha}\right\}_{\alpha \in A}$. Then the set $D$ of points of discontinuity of $f$ can be represented by

$$
D=\bigcup_{\alpha \in A} f^{-1}\left(U_{\alpha}\right) \backslash \text { int } f^{-1}\left(U_{\alpha}\right) .
$$

Proof. If $x$ is a discontinuous point of $f$, then there exists an open neighbourhood $V(y)$ of $y=f(x)$ such that $x$ is not an interior point of $f^{-1}(V(y))$, i.e. $x \in f^{-1}(V(y)) \backslash$ int $f^{-1}(V(y))$. Since $\left\{U_{\alpha}\right\}$ is a base of $Y$, we can suppose that $U_{\alpha} \subset V(y)$ for some $\alpha$. Hence, $x \in \bigcup_{\alpha \in A} f^{-1}\left(U_{\alpha}\right) \backslash$ int $f^{-1}\left(U_{\alpha}\right)$. Conversely, if for $x \in X$, there exists $\alpha \in A$ such that $x \in f^{-1}\left(U_{\alpha}\right) \backslash$ int $f^{-1}\left(U_{\alpha}\right)$, then $x$ is not an interior point of the inverse image of an open neighbourhood $U_{\alpha}$ of $f(x)$, and so $f$ is not continuous at $x$.

Proposition 3.2. Let $X$ be a topological space, $Y=(Y, d)$ be a metric space and $f: X \rightarrow Y$ be a mapping. The set $D$ of points of discontinuity of $f$ is an $F_{\sigma}$ set in $X$.

Proof. Suppose that for any $x \in X,\left\{V_{\beta}(x)\right\}$ is a base of neighbourhoods of $x$. Define the oscillation function $\omega: X \rightarrow \mathbf{R}_{+}$of $f$ as follows:

$$
\forall x \in X, \quad \omega(x)=\lim _{\beta} \sup _{x_{1}, x_{2} \in V_{\beta}(x)} d\left(f\left(x_{1}\right), f\left(x_{2}\right)\right) .
$$

It is easy to verify that $\omega$ is a upper semi-continuous function, i.e. for $c \geq 0$, $\omega^{-1}([c,+\infty))$ is closed in $X$. Since

$$
D=\{x \in X \mid \omega(x) \geq 1 / n, \text { for some } n \geq 1\}=\bigcup_{n=1}^{\infty} \omega^{-1}([1 / n,+\infty)),
$$

it is an $F_{\sigma}$ set.

Proposition 3.3. Let $X$ and $Y$ be two topological spaces, $Y$ satisfy the second axiom of countability and $f: X \rightarrow Y$ be a mapping. If for each open set $U_{Y}$ of $Y, f^{-1}\left(U_{Y}\right)$ is the union of an open set and a nowhere dense set in $X$, then the set of points of discontinuity of $f$ is of the first category.

Proof. Since $Y$ has a countable base, we can suppose that $\left\{U_{k}\right\}_{k=1,2, \ldots}$ is a base of $Y$. According to Proposition 3.1, the set of points of discontinuity of $f$ is

$$
D=\bigcup_{k=1}^{\infty} f^{-1}\left(U_{k}\right) \backslash \text { int } f^{-1}\left(U_{k}\right)
$$

From the assumptions of the proposition, $f^{-1}\left(U_{k}\right) \backslash$ int $f^{-1}\left(U_{k}\right)$ is nowhere dense. Hence, $D$ is of the first category. 
Proposition 3.4. Let $X$ and $Y$ be two topological spaces, $f: X \rightarrow Y$ be robust and $S \subset X$ be the set of points of continuity of $f$. If $S$ is dense in $X$, then $f$ is approximatable.

Proof. We have to show that for any $x \in X$ and $y=f(x) \in Y$, there exists a net $\left\{x_{\alpha}\right\} \subset S$ such that

$$
\lim _{\alpha} x_{\alpha}=x \text { and } \lim _{\alpha} f\left(x_{\alpha}\right)=f(x) .
$$

Suppose that $\left\{V_{\beta}(x)\right\}$ and $\left\{U_{y}(y)\right\}$ are open neighbourhood bases of $x$ in $X$ and of $y$ in $Y$, respectively. Then for any $\beta$ and any $\gamma, f^{-1}\left(U_{\gamma}(y)\right) \cap V_{\beta}(x)$ is a nonempty robust set in $X$; in particular, its interior is nonempty. Since $S$ is dense in $X$, we have that

$$
\forall \beta, \gamma, \quad \exists x_{\beta \gamma} \in f^{-1}\left(U_{\gamma}(y)\right) \cap V_{\beta}(x) \cap S .
$$

Set $\alpha=(\beta, \gamma)$ and $x_{\alpha}=x_{\beta \gamma}$. Then (10) holds, where $\alpha=(\beta, \gamma) \succ \alpha^{\prime}=$ $\left(\beta^{\prime}, \gamma^{\prime}\right)$ is defined by $V_{\beta}(x) \subset V_{\beta^{\prime}}(x)$ and $U_{y}(y) \subset U_{\gamma^{\prime}}(y)$.

Proof of Theorem 3.1. From Propositions 2.1 and 3.3, the set $D$ of points of discontinuity of $f$ is of the first category. Since $X$ is a Baire space, the set of points of continuity of $f, X \backslash D$, is dense in $X$. Joining this up with Propositions 3.2 and 3.4 proves the theorem.

The following two examples show that the assumptions on $X$ and $Y$ in Theorem 3.1 are indispensable.

Example 3.1. Let $X=\left\{r_{k}\right\}=$ set of all the rational numbers in $[0,1]$ with the induced topology by $[0,1]$ (so $X$ is not a Baire space), $Y=[0,1]$ and $\alpha_{k}>0, k=1,2, \ldots$, with $\sum_{k=1}^{\infty} \alpha_{k}=1$. Then the function

$$
f(x)=\sum_{r_{k}<x} \alpha_{k}
$$

is robust, but discontinuous at every point in $X$.

Example 3.2. Let $X=\mathbf{R}$ and $Y=\mathbf{R}^{\mathbf{R}}$ with the product topology. $Y$ can be identified with a space of all functions $g(\cdot): \mathbf{R} \rightarrow \mathbf{R}$ with the topology of the pointwise convergence. This topological space has no countable base. Let $f: X \rightarrow Y$ be defined as follows.

$$
f(x)(z)=g_{x}(z)= \begin{cases}1 & \text { if } z \geq x \\ 0 & \text { if } z<x\end{cases}
$$

The sets of the following form in $Y$ constitute a base of the open neighbourhoods of $y=f(x)$ :

$$
U\left(y ; z_{1}, \ldots, z_{k} ; \varepsilon\right)=\left\{h(\cdot) \in Y|| g_{x}\left(z_{i}\right)-h\left(z_{i}\right) \mid<\varepsilon, i=1,2, \ldots, k\right\}
$$

where $z_{i} \in \mathbf{R}, i=1,2, \ldots, k$ and $\varepsilon \in(0,1)$. Since

$$
\begin{aligned}
f^{-1}(U(y ; z ; \varepsilon)) & =\left\{x^{\prime} \in \mathbf{R}|| g_{x^{\prime}}(z)-g_{x}(z) \mid<\varepsilon\right\} \\
& = \begin{cases}(z,+\infty) & \text { if } z<x, \\
(-\infty, z] & \text { if } z \geq x,\end{cases}
\end{aligned}
$$


we have that

$$
\begin{aligned}
f^{-1}\left(U\left(y ; z_{1}, \ldots, z_{k} ; \varepsilon\right)\right)=\left\{x^{\prime} \in \mathbf{R} \| g_{x^{\prime}}\left(z_{i}\right)-g_{x}\left(z_{i}\right) \mid<\varepsilon, i=1, \ldots, k\right\} \\
=\left\{\begin{array}{l}
\left(\max _{i} z_{i},+\infty\right) \text { if } \max _{i} z_{i}<x, \\
\left(-\infty, \min _{i} z_{i}\right] \text { if } \min _{i} z_{i} \geq x, \\
\left(z_{i}, z_{i+1}\right] \text { if } z_{1}<\cdots<z_{i}<x \leq z_{i+1}<\cdots<z_{k}
\end{array}\right.
\end{aligned}
$$

in which $x$ is a robust point. Hence, $f$ is a robust mapping from $X$ to $Y$.

$f: \mathbf{R} \rightarrow \mathbf{R}^{\mathbf{R}}$ is continuous at $x \in \mathbf{R}$ iff

$$
\forall z \in \mathbf{R}, \quad \lim _{x^{\prime} \rightarrow x} g_{x^{\prime}}(z)=g_{x}(z) .
$$

Since we have always that

$$
\forall x \in \mathbf{R}, \quad \limsup _{x^{\prime} \rightarrow x} g_{x^{\prime}}(x)=1 \text { and } \liminf _{x^{\prime} \rightarrow x} g_{x^{\prime}}(x)=0,
$$

$f$ is discontinuous at all $x \in \mathbf{R}$.

Remark. Recall ([3], [4], [6]) that a mapping $f: X \rightarrow Y$ is said to be of the first Baire class iff for every open set $U_{Y}$ of $Y, f^{-1}\left(U_{Y}\right)$ is an $F_{\sigma}$ set. When $X=Y=\mathbf{R}$, every function from $\mathbf{R}$ to $\mathbf{R}$ of the first Baire class is measurable. A classic theorem says that under the hypotheses in Theorem 3.1, the set of points of continuity of a mapping of the first Baire class is a dense $G_{\delta}$ set. However, a robust mapping may not be of the first Baire class and vice versa. For instance, the function in (2) is of the first Baire class, because it is lower semi-continuous and every l.s.c. function is of the first class; an example of a nonmeasurable robust real function (then, it is impossible to be a function of the first Baire class) is as follows.

Example 3.3. Let $X=[0,1]$ and $C \subset[0,1]$ be a Cantor-type set with positive Lebesgue measure, which is a nowhere dense perfect set (no isolated points and closed). Then $X \backslash C$ is open. Take a non-measurable subset $C_{1}$ of $C$. Such a subset exists [6]. Divide $X$ into two disjoint robust parts $A_{1}$ and $A_{2}$ such that $C_{1} \subset A_{1} \backslash$ int $A_{1}$ and $C \backslash C_{1} \subset A_{2} \backslash$ int $A_{2}$. Then $A_{1}$ and $A_{2}$ are also nonmeasurable. The characteristic function of $A_{1}$ :

$$
\chi_{A_{1}}(x)= \begin{cases}1 & \text { if } x \in A_{1}, \\ 0 & \text { if } x \notin A_{1},\end{cases}
$$

is piecewise continuous, but nonmeasurable. The construction of $A_{1}, A_{2}, C_{1}$ and $C_{2}$ is similar to that in [14].

\section{BANACH SPACES OF BOUNDED ROBUST MAPPINGS}

In this section, we assume that $X$ is a complete metric space and $Y$ is a real separable Banach space. Then the hypotheses in Theorem 3.1 hold and all the robust mappings from $X$ to $Y$ are approximatable. We will discuss the construction of the set of all the bounded robust mappings in this setting.

Since $Y$ is a real Banach space, we can consider linear operations for mappings from $X$ to $Y$. If $f: X \rightarrow Y$ is robust, then for any $\lambda \in \mathbf{R}, \lambda f$, defined by

$$
\forall x \in X, \quad(\lambda f)(x)=\lambda f(x)
$$


is also robust. But, if $f_{1}$ and $f_{2}$ are robust, then, in general, $f_{1}+f_{2}$, defined by

$$
\forall x \in X, \quad\left(f_{1}+f_{2}\right)(x)=f_{1}(x)+f_{2}(x),
$$

is not necessarily robust.

Example 4.1. Let $X=Y=\mathbf{R}$,

$$
f_{1}(x)= \begin{cases}1 & \text { if } x>0 \\ 0 & \text { if } x \leq 0\end{cases}
$$

and

$$
f_{2}(x)= \begin{cases}1 & \text { if } x<0 \\ 0 & \text { if } x \geq 0\end{cases}
$$

Then $f_{1}$ and $f_{2}$ are robust. But

$$
\left(f_{1}+f_{2}\right)(x)= \begin{cases}1 & \text { if } x \neq 0 \\ 0 & \text { if } x=0\end{cases}
$$

is not robust.

This example is typical. By the same idea we can claim that for any discontinuous robust mapping $f$, there always exists another discontinuous robust mapping $g$ such that $f+g$ is not robust.

In this section, we will look for a condition for the robustness of the sum of two robust mappings, or a condition under which a family of robust mappings becomes a linear or Banach space.

Definition 4.1. Let $S \subset X$ be dense in $X$,

$$
c_{S}=\left\{\left\{s_{k}\right\} \subset S \mid \exists x \in X, \lim _{k \rightarrow \infty} s_{k}=x\right\}
$$

and $A_{S}: X \backslash S \rightarrow c_{S}$ such that for any $x \in X \backslash S, A_{S}(x)=\left\{s_{k}(x)\right\}$ satisfies $\lim _{k \rightarrow \infty} s_{k}(x)=x$. If $f: X \rightarrow Y$ possesses the following property:

1. $f$ is continuous at each point $s \in S$;

2. for any $x \in X \backslash S$ and $A_{S}(x)=\left\{s_{k}(x)\right\} \subset S, f(x)=\lim _{k \rightarrow \infty} f\left(s_{k}(x)\right)$; then we say that $f$ belongs to the $\left(S, A_{s}\right)$-class. The set of all the mappings of the $\left(S, A_{S}\right)$-class is denoted by $F_{\left(S, A_{S}\right)}(X, Y)$.

Obvously, each mapping of an ( $\left.S, A_{S}\right)$-class is robust (approximatable) and each robust mapping belongs to a certain $\left(S, A_{S}\right)$-class, but not uniquely. Any continuous mapping belongs to all the $\left(S, A_{S}\right)$-classes and, in particular, all the continuous mappings from $F_{\left(X, A_{X}\right)}=F_{(X, \varnothing)}=C(X, Y)$.

Proposition 4.1. 1. If $f_{1}, f_{2} \in F_{\left(S, A_{S}\right)}(X, Y)$, then for any $\lambda_{1}, \lambda_{2} \in \mathbf{R}, \lambda_{1} f_{1}+$ $\lambda_{2} f_{2} \in F_{\left(S, A_{S}\right)}(X, Y)$.

2. If $\left\{f_{n}\right\} \subset F_{\left(S, A_{S}\right)}(X, Y)$ and for a mapping $f: X \rightarrow Y$,

$$
\lim _{n \rightarrow \infty} \sup _{x \in X}\left\|f_{n}(x)-f(x)\right\|=0,
$$

then $f \in F_{\left(S, A_{S}\right)}(X, Y)$.

The proof is elementary.

Set

$$
B_{\left(S, A_{S}\right)}(X, Y)=\left\{f \in F_{\left(S, A_{S}\right)}(X, Y) \mid \sup _{x \in X}\|f(x)\|<+\infty\right\}
$$


Then Proposition 4.1 means that defining the norm $\|f\|=\sup _{x \in X}\|f(x)\|$, $B_{\left(S, A_{S}\right)}(X, Y)$ is a Banach space. $B_{\left(S, A_{S}\right)}(X, Y)$ includes the Banach space of bounded continuous mappings from $X$ to $Y$, denoted by $B_{C}(X, Y)$, as a closed subspace. On the other hand, each bounded robust mapping from $X$ to $Y$ belongs to some $B_{\left(S, A_{S}\right)}(X, Y)$. Denoting the set of all the bounded robust mappings from $X$ to $Y$ by $B_{R}(X, Y)$, we have that

$$
\bigcup_{\left(S, A_{S}\right)} B_{\left(S, A_{S}\right)}(X, Y)=B_{R}(X, Y) \text { and } \bigcup_{\left(S, A_{S}\right)} B_{\left(S, A_{S}\right)}(X, Y)=B_{C}(X, Y) \text {. }
$$

Furthermore, if for two dense sets $S_{1}$ and $S_{2}$ in $X$ and for two mappings $A_{S_{1}}: X \backslash S_{1} \rightarrow c_{S_{1}}$ and $A_{S_{2}}: X \backslash S_{2} \rightarrow c_{S_{2}}$, we have that

$$
S_{1} \subset S_{2} \text { and } \forall x \in X \backslash S_{2}, \quad A_{S_{1}}(x) \subset A_{S_{2}}(x),
$$

then it follows that

$$
B_{\left(S_{1}, A_{S_{1}}\right)}(X, Y) \supset B_{\left(S_{2}, A_{S_{2}}\right)}(X, Y) .
$$

However, any $B_{\left(S, A_{S}\right)}(X, Y)$ is not maximal with respect to the partial ordering of set inclusion, because for any $B_{\left(S, A_{S}\right)}(X, Y)$, it is easy to construct another $B_{\left(S^{\prime}, A_{S^{\prime}}^{\prime}\right)}$ which contains $B_{\left(S, A_{S}\right)}$ as a proper subclass.

Let $M(X, Y)$ be the set of all Banach spaces of bounded robust mappings from $X$ to $Y$. We can define the set inclusion as a partial order relation in $M(X, Y)$. This partial order in $M(X, Y)$ is inductive, i.e. each totally ordered subset of $M(X, Y)$ has a maximal element, which is the completion of the union of all the elements (considered as the subsets of $B_{R}(X, Y)$ ) of this totally ordered subset. Thanks to Proposition 2.2, it is easy to show that the uniform limit of a sequence of bounded robust mappings is also a bounded robust mapping. Hence, by Zorn's lemma, $M(X, Y)$ possesses maximal elements, which are the "maximal Banach spaces" in $B_{R}(X, Y)$. Denote these "maximal Banach spaces" of bounded robust mappings from $X$ to $Y$ by $B_{M \delta}(X, Y), \delta \in \Delta$. Then we have that

$$
\bigcup_{\delta \in \Delta} B_{M \delta}(X, Y)=B_{R}(X, Y) \text { and } \bigcap_{\delta \in \Delta} B_{M \delta}(X, Y)=B_{C}(X, Y) \text {. }
$$

If $B_{M \delta_{1}}(X, Y) \neq B_{M \delta_{2}}(X, Y)$, then they will not be included in each other.

It is interesting to investigate the quotient space of $B_{\left(S, A_{S}\right)}$ to $B_{C}(X, Y)$. Even for $X=[0,1], Y=\mathbf{R}$ and $X \backslash S$ a singleton, the quotient space $B_{\left(S, A_{S}\right)}([0,1], \mathbf{R}) / B_{C}([0,1], \mathbf{R})$ is not trivial.

Finally, if $Y$ is a separable Banach algebra, then any $B_{\left(S, A_{S}\right)}$ is also a Banach algebra by defining

$$
\forall x \in X, \quad\left(f_{1} f_{2}\right)(x)=f_{1}(x) f_{2}(x) .
$$

The similar conclusion for the "Banach algebras of bounded robust mappings" also holds.

\section{ROBUST FUNCTIONS}

Now we assume that $X$ is a Baire space and $Y=\mathbf{R}$. In this case, the robust mappings become the "robust functions", which have a set of points of continuity dense in $X$ and are approximatable at their points of discontinuity. 
However, it is not necessary to only consider robust functions with respect to the natural topology of $\mathbf{R}$. In fact, in Zheng [10]-[12], for the application to the global minimization problem, a robust function is always defined for the "upper topology" of $\mathbf{R}$, which means that all the open sets have a form of $(-\infty, c)$, $c \in \mathbf{R}$. To distinguish robust functions with respect to the diverse topologies of $\mathbf{R}$, we propose the following definitions.

Definition 5.1. Let $X$ be a topological space and $f: X \rightarrow \mathbf{R} . \quad f$ is said to be robust (respectively upper robust or lower robust) at $x$ iff for any $\varepsilon>0, f^{-1}((f(x)-\varepsilon, f(x)+\varepsilon))$ (respectively, $f^{-1}((-\infty, f(x)+\varepsilon)$ ) or $\left.f^{-1}((f(x)-\varepsilon,+\infty))\right)$ is a semi-neighbourhood of $x . f$ is said to be a robust function (respectively, upper or lower robust function) iff $f$ is robust (respectively, upper or lower robust) at all $x \in X$; or for any $a, b \in \mathbf{R}$ (respectively, $c \in \mathbf{R}), f^{-1}((a, b))$ (respectively, $f^{-1}((-\infty, c))$ or $f^{-1}((c,+\infty))$ ) is robust in $X$.

The concepts of robustness, upper robustness and lower robustness for a function are extensions of continuity, upper semi-continuity and lower semicontinuity. Any continuous (respectively, upper or lower semi-continuous) function is robust (respectively, upper or lower robust), but a robust (respectively, upper or lower robust) function may not be continuous (respectively, upper or lower semi-continuous). In addition, an upper robust and lower semi-continuous (respectively, a lower robust and upper semi-continuous) function is robust, but an upper and lower robust function may not be robust, because the intersection of two semi-neighbourhoods may not be a semi-neighbourhood.

Example 5.1. Let $X=\mathbf{R}$ and $f$ be defined by

$$
f(x)= \begin{cases}1 & \text { if } x>0 \\ 0 & \text { if } x=0 \\ -1 & \text { if } x<0\end{cases}
$$

Then $f$ is neither upper or lower semi-continuous nor robust, but is both upper and lower robust.

From Proposition 2.2, we obtain

Proposition 5.1. Let $X$ be a topological space and $f_{\delta}: X \rightarrow \mathbf{R}, \delta \in \Delta$, be a net of functions. If all the functions $f_{\delta}$ are robust at $\bar{x} \in X$ and a function $f: X \rightarrow \mathbf{R}$ satisfies

$$
\lim _{\delta} \sup _{x \in X}\left|f_{\delta}(x)-f(x)\right|=0,
$$

then $f$ is also robust at $\bar{x}$.

Proposition 2.2 is not applicable for the upper or lower robustness of functions, because $\mathbf{R}$ with the "upper" or "lower" topology is not a metric space, not even a Hausdorff space. But it is obvious that Proposition 5.1 is also valid for upper or lower robust functions. However, for these two cases, we can modify the concept of "uniform convergence" and the following proposition is valid:

Proposition 5.2. Let $X$ be a topological space, $f_{\delta}: X \rightarrow \mathbf{R}, \delta \in \Delta$, be a net of functions and all the function $f_{\delta}$ be upper (respectively, lower) robust at $\bar{x} \in X$. 
If for a function $f: X \rightarrow \mathbf{R}$ and for all $x \in X$, we have uniformly

$$
\liminf _{\delta} f_{\delta}(x) \geq f(x) \quad\left(\text { resp. } \limsup _{\delta} f_{\delta}(x) \leq f(x)\right)
$$

and

$$
\lim _{\delta} f_{\delta}(\bar{x})=f(\bar{x}),
$$

then $f$ is also upper (respectively, lower) robust at $\bar{x}$.

Proof. We have to show that for $\bar{x} \in X$ and any $\varepsilon$-"upper neighbourhood" of $\bar{y}=f(\bar{x}), U_{\varepsilon}(f(\bar{x}))=(-\infty, f(\bar{x})+\varepsilon)(\varepsilon>0), f^{-1}\left(U_{\varepsilon}(f(\bar{x}))\right)$ is a semineighbourhood of $\bar{x}$. Thus, we need to show that there exists a semi-neighbourhood of $\bar{x}, V(\bar{x})$, such that

$$
\forall x \in V(\bar{x}), \quad f(x)<f(\bar{x})+\varepsilon .
$$

From (14), using standard $\varepsilon / 3$ argument, we can verify easily that (16) holds.

In the case of the lower robust functions, the proof is similar.

Proposition 5.3. Let $X$ be a topological space, $f_{\gamma}: X \rightarrow \mathbf{R}, \gamma \in \Gamma$, be a family of functions and all the function $f_{y}$ be upper (resp., lower) robust at $\bar{x} \in X$. If for a function $f: X \rightarrow \mathbf{R}$ and for all $x \in X, f(x)=\inf _{y \in \Gamma} f_{\gamma}()$ (resp., $\left.f(x)=\sup _{y \in \Gamma} f_{\gamma}(x)\right)$, then $f$ is also upper (resp., lower) robust at $\bar{x}$.

Now we apply Theorem 3.1 to the case of robust functions.

Theorem 5.1. Let $X$ be a complete metric space, $f: X \rightarrow \mathbf{R}$ be a function on $X$ and $S$ be the set of points of continuity of $f$. Then $f$ is robust iff $f$ is approximatable, i.e. $S$ is dense in $X$ and for any $x \in X \backslash S$ there exists $a$ sequence $\left\{s_{k}(x)\right\} \subset S$ such that $\lim _{k \rightarrow \infty} f\left(s_{k}(x)\right)=f(x)$. In this case, $S$ is a dense $G_{\delta}$ set in $X$.

Although $\mathbf{R}$ with the upper or lower topology is not a Hausdorff space, the second axiom of countability is valid. So, replacing the continuity by the upper or lower semi-continuity, a similar theorem for an upper or a lower robust function is also valid. However, we can prove a stronger theorem as follows.

Theorem 5.2. Let $X$ be a complete metric space, $f: X \rightarrow \mathbf{R}$ be a function on $X$ and $S$ be the set of points of continuity of $f$. Then $f$ is upper (respectively, lower) robust iff $S$ is dense in $X$ and for any $x \in X \backslash S$, there exists a sequence $\left\{s_{k}(x)\right\} \subset S$ such that $\lim _{\sup _{k \rightarrow \infty}} f\left(s_{k}(x)\right) \leq f(x)$ (respectively, $\left.\liminf _{k \rightarrow \infty} f\left(s_{k}(x)\right) \geq f(x)\right)$. In this case, $S$ is a dense $G_{\delta}$ set in $X$.

Proof. Consider an upper robust function $f$. Let $\left\{r_{k}\right\}$ be the set of rational numbers in $\mathbf{R}$. We prove that

$$
D=\bigcup_{i \neq j} f^{-1}\left(\left(r_{i}, r_{j}\right)\right) \backslash \text { int } f^{-1}\left(\left(r_{i}, r_{j}\right)\right)
$$

is of the first category. It suffices to show that $f^{-1}((a, b)) \backslash$ int $f^{-1}((a, b))$ is of the first category for any $a, b \in \mathbf{R}$.

Since $f$ is upper robust, $f^{-1}([c,+\infty))=X \backslash f^{-1}((-\infty, c))$ is the complement of a robust set. From Proposition 2.1, it is the union of an open set and 
a nowhere dense set. Hence,

$$
\begin{aligned}
f^{-1}((a, b)) & =f^{-1}((-\infty, b)) \cap f^{-1}((a,+\infty)) \\
& =f^{-1}((-\infty, b)) \cap\left(\bigcup_{n=1}^{\infty} f^{-1}([a+(1 / n),+\infty))\right) \\
& =V \cap\left(\bigcup_{n=1}^{\infty}\left[O_{n} \cup T_{n}\right]\right),
\end{aligned}
$$

where $V$ is a robust set, $O_{n}$ is open and $T_{n}$ is nowhere dense, $n=1,2, \ldots$. Thus, we have that

$$
f^{-1}((a, b))=\bigcup_{n=1}^{\infty}\left[\left(V \cap O_{n}\right) \cup\left(V \cap T_{n}\right)\right]=\bigcup_{n=1}^{\infty}\left(V_{n} \cup T_{n}^{\prime}\right),
$$

where $V_{n}$ is robust (maybe empty) and $T_{n}^{\prime}$ is nowhere dense, $n=1,2, \ldots$; and

$$
f^{-1}((a, b)) \backslash \text { int } f^{-1}((a, b)) \subset \bigcup_{n=1}^{\infty}\left(V_{n} \backslash \text { int } V_{n}\right) \cup T_{n}^{\prime} .
$$

The right side of this inclusion is obviously of the first category, and so is the left side. The $G_{\delta}$-ness of $S$ is a consequence of Proposition 3.2.

In Example 2.1, if $\alpha \geq-1$, then $f$ is upper robust; and if $\alpha \leq 1$, then $f$ is lower robust.

Finally, the discussion of the last section is suitable to the robust functions, but not to the upper or lower robust functions. If $f$ is upper (respectively, lower) robust, then only for any $\lambda \geq 0, \lambda f$ is also upper (respectively, lower) robust, but for any $\lambda \leq 0, \lambda f$ is lower (respectively, upper) robust. However, we have the similar conclusion for the upper or lower robustness of the sum of two upper or lower robust functions.

\section{Conclusions}

The initial motivation to introduce the robustness of a set or of a function ([10]-[12]) is to enlarge the class of objective functions and of constraint sets of global optimization problems. This paper demonstrates that the concept of robustness is essential for numerical analysis. We show that if $X$ is a complete metric space and $Y$ has a countable base, then a robust mapping $f: X \rightarrow Y$ is precisely an approximatable mapping. For the problem of solving equation (1), we only need $Y$ to be a metric space $(Y, d)$, which may not have a countable base. This is because the problem is equivalent to the following problem:

$$
\text { to find } \bar{x} \in X \text { such that } d(f(\bar{x}), \bar{y})=0 \text {. }
$$

If $f: X \rightarrow Y$ is a robust mapping, then it is obvious that the function $x \mapsto$ $d(f(x), \bar{y})$ from $X$ to $\mathbf{R}_{+}$(having a countable base) is also robust, and so, approximatable. Notice that the problem (20) is a global minimization problem.

In Zheng [10]-[12], for a global minimization problem, the objective function $f$ is always assumed to be lower semi-continuous and "upper robust" (according to our definition). Now we know that it is equivalent to assume that $f$ is l.s.c. 
and robust, or $f$ is a l.s.c. approximatable function. In general, a l.s.c. function is not approximatable. (2) is a typical example.

In Chew-Zheng [2], Zheng [11] and other works, a theory of integral global optimization is presented. In this theory, we require an objective function to be integrable and robust. In general, a robust function may not be integrable. Now we know that the set of points of discontinuity of a robust function is always an $F_{\sigma}$ set of the first category. When $X$ is $[0,1]^{k}$, it is reasonable to assume that this $F_{\sigma}$ set of the first category is a Lebesgue null set, and then a robust function becomes a Riemann integrable function. In particular, when $k=1$, a robust function will be topologically equivalent to a Riemann integral function [6, Theorem 13.3]. This situation is very favourable to the theory of integral global optimization.

In the applications of integral global optimization, we often require the sum of two robust functions to be robust. Before this paper, we only knew that it suffices to require one of the functions to be continuous. Now we have indeed a necessary and sufficient condition for the robustness of the sum of two robust functions; that is, they must possess the same property of discontinuity.

In [2], [11] and others, a Monte Carlo implementation of the integral global minimization algorithm was also proposed. It is shown that the global minimizers can be obtained with high probability. Due to the stochastic nature of Monte Carlo implementation, one would suspect that some global minimizers may be lost during the course of computation. However, according to our computation experiences over the past twenty years, we can always find global minimizers even for discontinuous functions [2], [16]. The main theorem of this paper could give a new theoretical explanation of our method, but it would be the subject of another paper.

The main results in this paper can be generalized to the case of set-valued mappings. The reader is referred to [8] for details.

\section{ACKNOWLEDGEMENT}

The authors would like to thank the anonymous referee for the very careful review and for many useful comments that improve the final version of the paper.

\section{REFERENCES}

1. L. V. Batuchtin and L. A. Maoboroda, Optimization of discontinuous functions, "Nauka", Moscow, 1984.

2. Soo Hong Chew and Quan Zheng, Integral global optimization: Theory, implementation and applications, Lecture Notes in Econ. and Math. Sys., vol. 298, Springer-Verlag, 1988.

3. G. Choquet, Outils topologiques et métriques de l'analyse mathématique, Centre de Documentation Universitaire et S. E. D. E. S. Réunis, Paris-V, 1969.

4. __ Lectures on analysis, Vol. 1, Benjamin, 1969.

5. I. V. Mayurova and R. G. Strongin, Minimization of multi-extremum function with a discontinuity, U.S.S.R. Comput. Math. and Math. Phys. 24 (1984), 121-126.

6. J. C. Oxtoby, Measure and category, 2nd ed., Springer-Verlag, 1980.

7. Z. Semadeni, Banach spaces of continuous functions, Polish Scientific Publishers, 1971.

8. Shuzhong Shi, Quan Zheng and Deming Zhuang, Set-valued robust mappings and approximatable mappings, J. Math. Anal. Appl. 183 (1994), 706-726.

9. I. Zang, Discontinuous optimization by smoothing, Math. Oper. Res. 6 (1981), 140-152. 
10. Quan Zheng, Robust analysis and global minimization of a class of discontinuous functions (I), Acta Math. Appl. Sinica (English Ser.) 6 (1990), 205-223.

11. _ Robust analysis and global minimization of a class of discontinuous functions (II), Acta Math. Appl. Sinica (English Ser.) 6 (1990), 317-337.

12. __ Robust analysis and global optimization, Comput. Math. Appl. 21 (1991), 17-24.

13. __ Global minimization of constrained problems with discontinuous penalty functions (to appear).

14. _. Discontinuity and measurability of robust functions in the integral global minimization, Comput. Math. Appl. 25 (1993), 79-88.

15. Quan Zheng and Deming Zhuang, Integral global optimization of constrained problems in functional space with discontinuous penalty functions, Recent Advances in Global Optimization (C. A. Floudas and P. M. Pardalos, eds.), Princeton Univ. Press, 1991.

16. Q. Zheng, B. Jiang and S. Zhuang, A method for finding global extrema, Acta. Math. Appl. Sinica 1 (1978), 161-174. (Chinese)

Nankai Institute of Mathematics, Tianjin 300071, China

E-mail address: shisz@bepc2.ihep.ac.cn

Department of Mathematics, Shanghai University, Shanghai 201800, China

Department of Mathematics and Computer Studies, Mount Saint Vincent University, Halifax, Nova Scotia, Canada B3M $2 \mathrm{~J} 6$

E-mail address: deming. zhuangemsvu.ca 